\title{
One Precursor but Two Types of Graphene Nanoribbons: On-Surface Transformations of 10,10'-Dichloro-9,9'-bianthryl on Ag(111)
}

\author{
Peter H. Jacobse, ${ }^{*}{ }^{\dagger}$ Konstantin A. Simonov, ${ }^{*}{ }^{\ddagger}$ Mark J. J. Mangnus, ${ }^{\dagger}$ Gleb I. Svirskiy, ${ }^{\S}$ \\ Alexander V. Generalov, \\ Alexei B. Preobrajenski, and Ingmar Swart \\ ${ }^{\dagger}$ Debye Institute for Nanomaterials Science, Utrecht University, PO Box 80000, 3508 TA Utrecht, The Netherlands \\ ${ }^{\ddagger}$ Department of Physics and Astronomy, Uppsala University, Box 516, 75120 Uppsala, Sweden \\ ${ }^{\S}$ V.A. Fock Institute of Physics, St. Petersburg State University, 198504 St. Petersburg, Russia \\ "MAX IV Laboratory, Lund University, Box 118, 22100 Lund, Sweden
}

Supporting Information

\begin{abstract}
On-surface synthesis has emerged in the last decade as a method to create graphene nanoribbons (GNRs) with atomic precision. The underlying premise of this bottomup strategy is that precursor molecules undergo a well-defined sequence of inter- and intramolecular reactions, leading to the formation of a single product. As such, the structure of the GNR is encoded in the precursors. However, recent examples have shown that not only the molecule, but also the coinage metal surface on which the reaction takes place, plays a decisive role in dictating the nanoribbon structure. In this work, we use scanning probe microscopy and X-ray photoelectron spectroscopy to investigate the behavior of 10,10'-dichloro-9,9'-bianthryl (DCBA) on $\mathrm{Ag}(111)$. Our

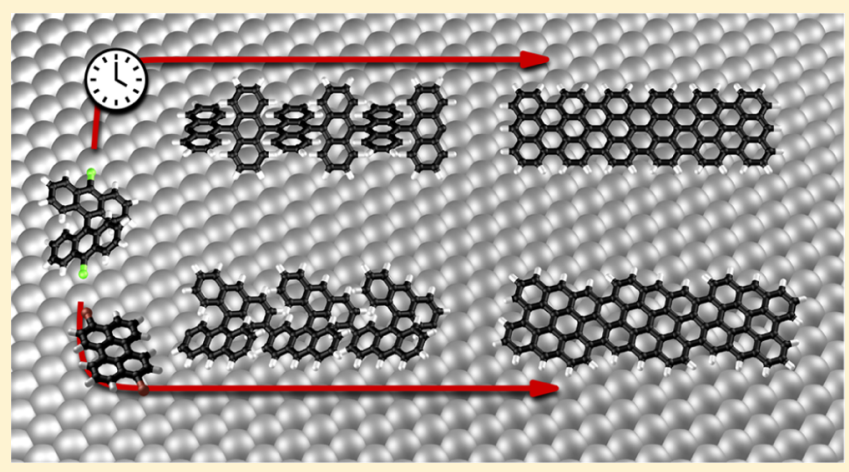
study shows that $\operatorname{Ag}(111)$ can induce the formation of both seven-atom wide armchair GNRs (7-acGNRs) and 3,1-chiral GNRs (3,1-cGNRs), demonstrating that a single molecule on a single surface can react to different nanoribbon products. We additionally show that coadsorbed dibromoperylene can promote surface-assisted dehydrogenative coupling in DCBA, leading to the exclusive formation of 3,1-cGNRs.
\end{abstract}

\section{INTRODUCTION}

Graphene nanoribbons (GNRs) are narrow strips of graphene that supplement the exceptional electronic properties of their two-dimensional parent material with a nonzero band gap by virtue of quantum confinement. ${ }^{1-8}$ A well-known quality of GNRs is that their electronic properties can be tailored by controlling their width and edge structure. ${ }^{1-4}$ During the last years, research has been focused on the synthesis of GNRs, ${ }^{9-20}$ ultimately envisioning their incorporation in future generations of (carbon-based) nanoelectronics. ${ }^{21-28}$ As structural irregularities inevitably lead to a poorly defined electronic structure (and resulting inefficiencies in electron transport due to defect scattering ${ }^{29-31}$ ), GNRs should be fabricated with atomic precision. This currently rules out top-down methodologies such as the etching of graphene or unzipping carbon nanotubes. Instead, ribbons can be produced with atomic precision via bottom-up strategies, most notably on-surface synthesis: a versatile method in which the structure is grown through polymerization reactions on single-crystal metal surfaces. Numerous types of GNRs have been fabricated using this methodology, with the geometric structure being encoded in the precursor molecule. ${ }^{19,32-40}$
So far, almost all on-surface syntheses of GNRs have relied on the Ullmann coupling reaction. Here, a halogen atom is dissociated from the aromatic precursor under the catalytic influence of the underlying substrate, leading to the formation of surface-stabilized radicals. The radical species then undergo intermolecular coupling to generate polymeric chains that can subsequently be converted into planar GNRs through cyclodehydrogenation (graphitization). Selecting a dedicated molecular precursor then gives a selective nanoribbon product. ${ }^{37,41}$ However, the paradigm "precursor molecule determines nanoribbon geometry" has recently been found to be deficient, as the first examples have emerged where different types of ribbons were obtained from a single molecule on different metal substrates. ${ }^{11,42,43}$ In particular, seven-atom wide armchair GNRs (7-acGNRs) can be prepared from $10,10^{\prime}$-dibromo-9,9' -bianthryl (DBBA) on $\mathrm{Au}(111),{ }^{10}$ whereas the anomalous 3,1-chiral GNR (3,1-cGNR) was formed when the synthesis was attempted on $\mathrm{Cu}(111){ }^{42,44}$ The same 3,1-

Received: December 19, 2018

Revised: February 19, 2019

Published: March 25, 2019 
cGNR was obtained using 10,10'-dichloro-9,9'-bianthryl (DCBA) - the chlorinated analogue of DBBA-and nonhalogenated 9,9'-bianthryl (BA) on $\mathrm{Cu}(111) .{ }^{45}$ Another surprising result is the formation of irregular polybisanthenes (irregular graphitic structures) when attempting on-surface synthesis with DCBA on $\mathrm{Au}(111){ }^{46}$ The result was rationalized by the fact that the $\mathrm{C}-\mathrm{Cl}$ bond is stronger than the $\mathrm{C}-\mathrm{Br}$ bond. ${ }^{47-49}$ As a consequence, for DCBA, the dehalogenation temperature is elevated relative to that for DBBA and is higher than the cyclodehydrogenation temperature. This leads to a reversed reaction sequence, with cyclodehydrogenation preceding covalent coupling.

From the above, it is clear that metal substrates catalyze various reactions in the formation process of GNRs: Ullmann coupling, surface-assisted dehydrogenative coupling, and dehalogenation. The exact sequence of thermally induced transformations is an interplay between the precursor molecule of interest and the metal substrate, and can additionally be affected by coadsorbed species. ${ }^{49-51}$ Understanding what product is obtained therefore requires an understanding of the various elementary reaction steps and their activation temperatures. Although scanning tunneling microscopy (STM) and noncontact atomic force microscopy (nc-AFM) imaging allow the identification of reaction products and intermediates, these techniques lack the ability to monitor chemical transformations at annealing temperatures. ${ }^{52,53}$ Recent studies have used X-ray photoelectron spectroscopy (XPS) to reveal activation temperatures of various elementary reaction steps in on-surface transformations. ${ }^{11,54-57}$ Scanning probe microscopy (SPM) and XPS can therefore be regarded as a complementary set of techniques to monitor the on-surface chemistry of molecular species: together, they provide a comprehensive understanding of the underlying processes.

In this work, we aim to complement our previous investigations of nanoribbon synthesis on the (111) facets of coinage metals (gold, silver, and copper) from dihalobianthryl precursors by exploring the behavior of DCBA on $\operatorname{Ag}(111)$. In addition, the behavior of nonhalogenated BA and DCBA with 3,9- and 3,10-dibromoperylene (DBP) was studied. The silver surface is interesting because it possesses intermediate reactivity with respect to the previously studied $\mathrm{Au}(111)$ and $\mathrm{Cu}(111)$ surfaces. As a result, the nature of the productpossibly the 7 -acGNR as on gold, ${ }^{46}$ the $3,1-\mathrm{cGNR}$ as on copper, ${ }^{45}$ or something different altogether-is difficult to predict a priori. To obtain a deeper understanding of the onsurface reaction sequence, we combined our STM studies with systematic, temperature-dependent XPS measurements, not only on $\operatorname{Ag}(111)$ but also on $\mathrm{Au}(111)$ and $\mathrm{Cu}(111)$. In the latter two cases, our work corroborates the earlier finding that, in contrast with what happens for DBBA, ${ }^{10,54}$ cyclodehydrogenation takes place before dehalogenation for DCBA on $\mathrm{Au}(111),{ }^{46}$ whereas non-Ullmann coupling occurs regardless of dehalogenation on $\mathrm{Cu}(111) .{ }^{45}$ In each case, we find that, for chlorinated molecules, the dehalogenation temperature is elevated compared to that for brominated precursors, which can crucially affect the sequence of on-surface reactions. In fact, we find that DCBA reacts on $\operatorname{Ag}(111)$ to form either the 7-acGNR or the 3,1-cGNR, whereas DBBA only gives 7acGNRs. ${ }^{58,59}$ Indeed, the higher dehalogenation temperature of DCBA (as compared to DBBA) increases the likelihood of switching to a non-Ullmann, surface-assisted dehydrogenative coupling mechanism. The surface-assisted dehydrogenative coupling pathway is furthermore selected when DCBA is annealed with co-adsorbed DBP, showing that the latter molecule can affect the reaction mechanism in such a way that coupling into 3,1-cGNRs is promoted. The exact atomic structure of the resulting GNRs and their polymeric intermediates were further resolved with STM and differential conductance mapping. Moreover, successive syntheses allowed us to produce armchair and chiral nanoribbons side-by-side, using just a single precursor species.

\section{METHODS}

Sample Preparation. DCBA was used as synthesized in our earlier work. ${ }^{46}$ BA, DBBA, and 3,(9/10)-DBP were also synthesized previously. ${ }^{38,60}$ Clean $\mathrm{Au}(111), \mathrm{Ag}(111)$, and $\mathrm{Cu}(111)$ surfaces were prepared by repeated cycles of $\mathrm{Ar}^{+}$ bombardment $\left(E_{\mathrm{Ar}^{+}}=1 \mathrm{keV}\right)$ at room temperatures (RTs) and subsequent annealing of the surface to $800 \mathrm{~K}$. The cleanliness of the substrate was verified either by XPS or by STM. Samples were prepared by vacuum sublimation of precursor molecules from an effusion cell onto the surface kept at RT. The thickness of deposited organics was calibrated by quartz microbalance, STM, and XPS measurements. The sample was gradually heated in ultra-high vacuum (UHV) to monitor the on-surface reactions.

STM Measurements. STM measurements were carried out in a Scienta Omicron variable-temperature STM/AFM instrument operated at $T=11.5 \mathrm{~K}$ under UHV conditions (base pressure $<10^{-9} \mathrm{mbar}$ ). Tip passivation was achieved by attachment of halogen atoms from the surface, similar to the procedure used to create CO-functionalized tips. ${ }^{61}$ Differential conductance mapping was performed in the constant current mode using a lock-in amplifier $\left[f=473 \mathrm{~Hz}, V_{\text {osc }}=20 \mathrm{mV}\right.$ $(\mathrm{rms})]$.

XPS and Near-Edge X-ray Absorption Fine Structure Measurements. XPS and near-edge X-ray absorption fine structure (NEXAFS) studies were performed at the HE-SGM beamline (bending magnet) at the HZB BESSY II synchrotron facility (Berlin, Germany). This beamline and its end station are specifically designed for studies of sensitive organic molecules and thin films. Photoelectron spectra were measured in normal emission (NE) geometry and normalized to the current in the storage ring and the number of scans. XPS measurements were performed at excitation energies of 300 and $380 \mathrm{eV}$, with a resolution of $\sim 0.3 \mathrm{eV}$. The binding energy scale of the spectra was referenced to the $\mathrm{Au} 4 \mathrm{f}_{7 / 2}$ emission at $84 \mathrm{eV}^{62}$ and (when possible) to the position of the Fermi level of the metal substrate. Peak-fitting analysis of the XPS spectra was performed using the XPS Tools (XPST) program package for IGOR Pro. ${ }^{63} \mathrm{C}$ K-edge NEXAFS spectra were recorded in the partial electron yield mode with a retarding voltage of $-150 \mathrm{eV}$. The angle between the polarization vector of X-rays and vector normal to the sample surface was either $55^{\circ}(\mathrm{NE})$ or $90^{\circ}$ (normal incidence geometry). Spectra were normalized to the corresponding spectra from the clean metal surfaces and to the continuum jump.

Tight-Binding Calculations. In some experiments, the structure of the GNR or polymer was inferred from STM imaging or differential conductance mapping. Tight-binding calculations were performed in order to correlate these experimental images with the correct structure. All calculations were performed using an on-site energy of $\varepsilon_{0}$ for the carbon $\mathrm{p}_{z}$ orbital and a nearest neighbor-hopping parameter $t$. Local density of states maps were generated by convolution of the 


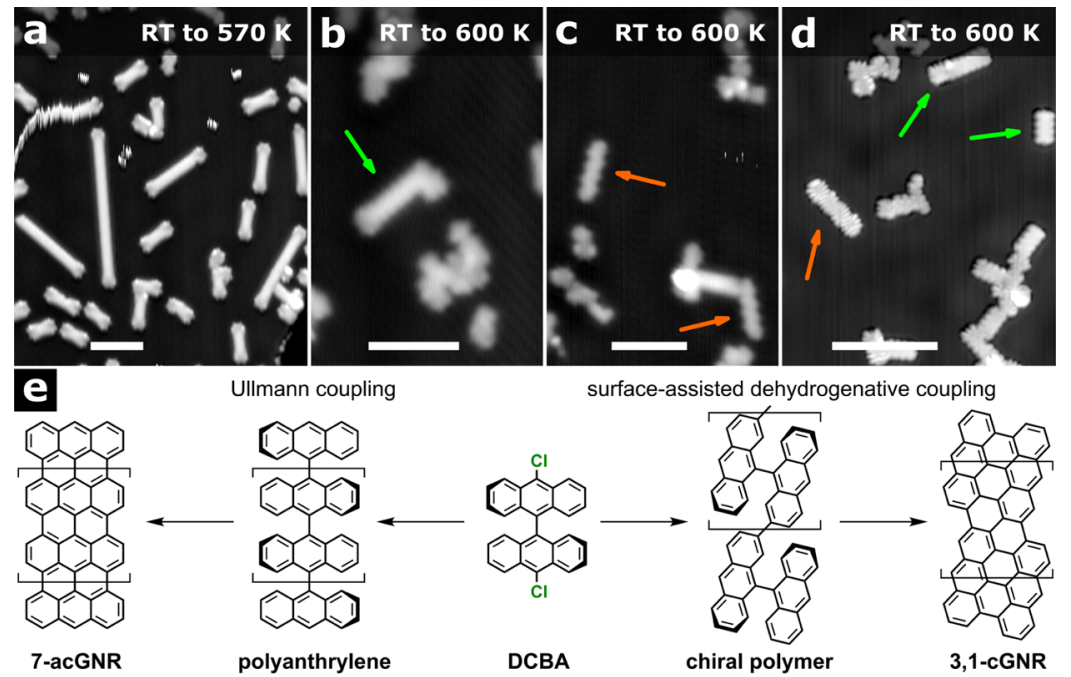

Figure 1. GNRs from DCBA on $\mathrm{Ag}(111)$. (a) STM scan of a sample prepared by annealing from RT to $570 \mathrm{~K}(\mathrm{~V}=-1 \mathrm{~V}, I=20 \mathrm{pA})$ showing armchair-type nanoribbons. (b) STM scan $(V=-1 \mathrm{~V}, I=20 \mathrm{pA})$ showing the result of annealing DCBA on $\mathrm{Ag}(111)$ to $600 \mathrm{~K}$, with an armchair nanoribbon indicated by the green arrow. (c) STM scan $(V=-1 \mathrm{~V}, I=20 \mathrm{pA})$ of another area of the sample of (b) where chiral nanoribbons (indicated by orange arrows) are found. (d) STM scan $(V=0.1 \mathrm{~V}, I=100 \mathrm{pA})$ of a sample prepared through codeposition of DBBA and DCBA and annealing to $600 \mathrm{~K}$, showing both 7-acGNRs (green arrows) and 3,1-cGNRs (orange arrow) lying side-by-side. (e) Mechanism of armchair nanoribbon formation from DCBA through Ullmann coupling (center to left) and of chiral nanoribbon formation from DCBA through the surfaceassisted dehydrogenative coupling mechanism (center to right). All scale bars are $5 \mathrm{~nm}$.

constituent orbitals with a tip of size $3 \AA$ and a Lorentzian energy broadening of the eigenstates by $36 \mathrm{mt}$.

\section{RESULTS AND DISCUSSION}

Nanoribbons from DCBA on Ag(111): STM Study. Figure 1a shows an STM scan of a $\operatorname{Ag}(111)$ surface onto which GNRs were synthesized through deposition of DCBA at RT followed by annealing at $570 \mathrm{~K}$. The temperature was ramped up slowly from RT to $570 \mathrm{~K}$ over the course of $20 \mathrm{~min}$. The STM topography shows GNRs with long, straight edges. The nanoribbons display distinct end-localized states, with the shape of "cat paws". These states become particularly noticeable when scanning at negative bias voltage. These features are consistent with 7 -acGNRs. ${ }^{64-68}$

Figure $1 b, c$ shows the results of deposition of DCBA at RT followed by rapid heating (instead of slow ramping) to $600 \mathrm{~K}$. Although difficult to quantify in our setup, the complete temperature ramp takes place within approximately $5 \mathrm{~min}$. Surprisingly, some areas on the crystal feature 7-acGNRs (Figure 1b), whereas in other areas, GNRs are seen with a characteristic sawtooth-like edge and without localized end states (Figure 1c). The appearance of these ribbons in STM is consistent with the 3,1-chiral GNR (3,1-cGNR). ${ }^{42,44,45,69}$ Consequently, we have managed to generate 7 -acGNRs and 3,1-cGNRs from the same precursor molecule on the same metal surface by simply varying the heating rate. The two different transformations-Ullmann coupling to give the 7acGNR and surface-assisted dehydrogenative coupling to give the 3,1-cGNR-are illustrated in Figure 1e. A rough estimate based on our STM images is that 3,1-cGNRs are approximately twice as predominant as 7-acGNRs, although the majority of the organic material has interconnected into undefined graphitic structures. Earlier studies have shown that 7-acGNRs can fuse laterally, after their initial formation, into 14-acGNRs and 21-acGNRs. The formation of 7-acGNRs and 3,1-cGNRs from DCBA, however, is to the best of our knowledge, the first instance where a single molecule, on a single metal surface, can give two types of nanoribbons, through two distinctly different and competing mechanisms.

In contrast, earlier studies have shown that the DBBA precursor yields only 7-acGNRs on $\operatorname{Ag}(111) \cdot{ }^{58,59,70}$ Figure S1 shows a reproduction of this experiment in our setup, where we resolve windmill-like self-assemblies of partially debrominated precursor molecules, the polyanthrylene intermediate, and the 7-acGNR product. While nanoribbon synthesis was also attempted with the nonhalogenated BA, this molecule was found to desorb from the surface at elevated temperatures, as evidenced by a completely empty surface in STM experiments. This finding is corroborated by an XPS experiment (see Figure S2). Similarly, thermal desorption (and absence of intermolecular coupling) was found for $\mathrm{BA}$ on $\mathrm{Au}(111){ }^{46}$

In an additional experiment, we codeposited equal amounts of DCBA and DBBA onto $\mathrm{Ag}(111)$. The deposition at RT was followed by quick heating to $600 \mathrm{~K}$. As seen in Figure 1d, both 7-acGNRs (indicated with green arrows) and 3,1-cGNRs (orange arrows) are produced. However, the two different types of GNRs are now mixed and not in separate domains. Assuming that the 7-acGNRs originate from DBBA, this shows another manifestation of the formation of 3,1-cGNR from DCBA. We therefore conclude that the $\operatorname{Ag}(111)$ surface can promote reaction pathways characteristic for both the less reactive $\mathrm{Au}(111)$ surface (formation of 7-acGNR via Ullmann coupling $)^{10,71}$ and the more reactive $\mathrm{Cu}(111)$ surface (formation of 3,1-cGNR via surface-assisted dehydrogenative coupling). ${ }^{42,44,45}$

We finalize this section by summarizing that DCBA can give both 7-acGNRs and 3,1-cGNRs on $\mathrm{Ag}(111)$. This anomalous behavior of DCBA shows that $\operatorname{Ag}(111)$ can promote the reaction pathways characteristic for both the more inert $\mathrm{Au}(111)^{10,71}$ (Ullmann coupling) and the more reactive $\mathrm{Cu}(111)^{42,44,45}$ (surface-assisted dehydrogenative coupling). This is, to the best of our knowledge, the first instance where a single molecule, on a single metal surface, can give two distinct types of nanoribbon products. At this point, we tentatively 

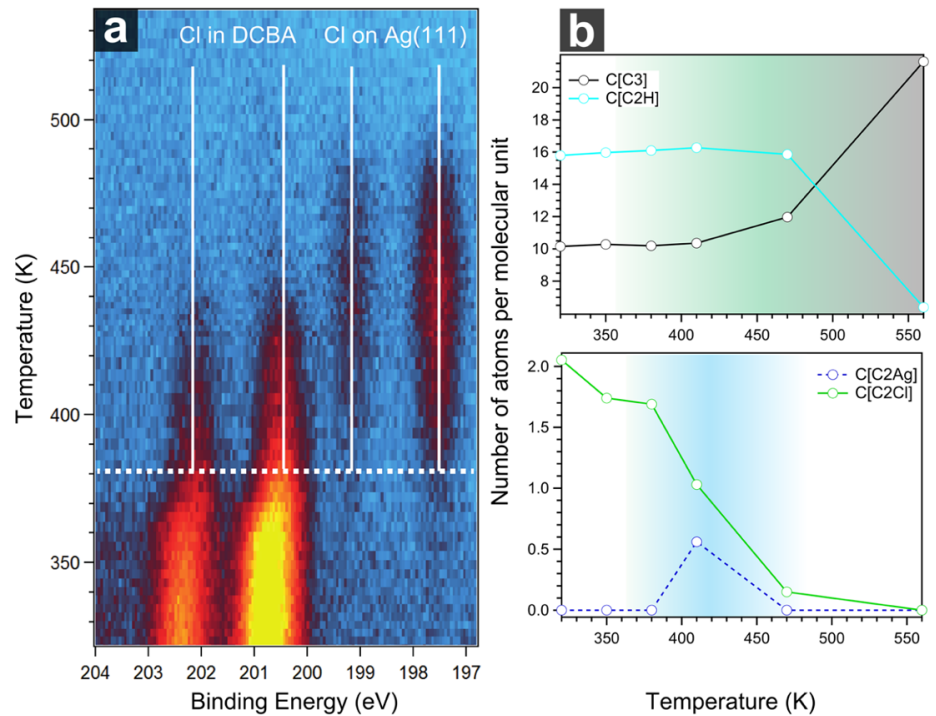

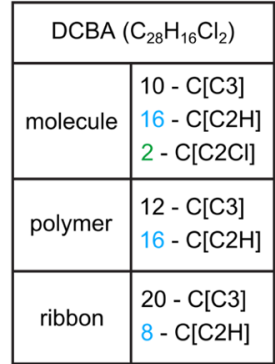

cyclodehydrogenation

dehalogenation

covalent coupling

Figure 2. XPS measurements of DCBA on $\mathrm{Ag}(111)$. (a) Evolution of the $\mathrm{Cl} 2 \mathrm{p}$ XPS spectrum for DCBA on $\mathrm{Ag}(111)$ mapped as a function of increasing sample temperature. The dashed line marks the beginning of the dechlorination process. The photon energy is $300 \mathrm{eV}$. The same color scale was used at all temperatures and the spectra were normalized to the ring current. (b) Results of the C 1s XPS peak fit analysis showing the changes in the number of carbon atoms in nonequivalent positions as calculated from the relative intensities of the corresponding $\mathrm{C} 1 \mathrm{~s}$ components. The different reaction steps are color-coded in accordance to the legend. The number of atoms in nonequivalent positions expected for one DCBA molecule and for a single repeating unit in a polymeric chain or GNR is shown on the right.

hypothesize that the formation of 3,1-cGNR is a consequence of blocking of the Ullmann coupling pathway, which could arise from passivation of bianthryl radical species by atoms on the surface. A deeper understanding of the processes at work comes from XPS experiments, which are discussed in the next section.

DCBA on Ag(111): XPS Study. The behavior of DCBA on $\operatorname{Ag}(111)$ was investigated in temperature-resolved XPS measurements, where both the $\mathrm{Cl} 2 \mathrm{p}$ and $\mathrm{C} 1 \mathrm{~s}$ lines were monitored. Figure $2 \mathrm{a}$ shows a $\mathrm{Cl} 2 \mathrm{p}$ map for DCBA on $\mathrm{Ag}(111)$, acquired by taking successive $\mathrm{Cl} 2 \mathrm{p}$ XPS spectra while heating the silver crystal from RT $(300 \mathrm{~K})$ to $560 \mathrm{~K}$ (at a heating rate of $3 \mathrm{~K} / \mathrm{min}$ ). This is the heating procedure found to yield 7-acGNRs exclusively. Studies of debromination of DBBA on metal surfaces, ${ }^{11,54,59}$ as well as deiodination of diiodoterphenyl ${ }^{57}$ indicate that dehalogenation is accompanied by the appearance of a new component in the halogen XPS spectra at lower binding energy $\left(E_{\mathrm{B}}\right)$. On this basis, the $\mathrm{Cl} 2 \mathrm{p}$ spin doublets centered around $E_{\mathrm{B}}\left(\mathrm{Cl} 2 \mathrm{p}_{3 / 2}\right)=200.7 \mathrm{eV}$ and around $E_{\mathrm{B}}\left(\mathrm{Cl} 2 \mathrm{p}_{3 / 2}\right)=197.5 \mathrm{eV}$ can be attributed to carbonbound chlorine in DCBA, and chlorine chemisorbed on $\mathrm{Ag}(111)$, respectively. The absence of chemisorbed chlorine at $\mathrm{RT}$ shows that the $\mathrm{C}-\mathrm{Cl}$ bond initially remains intact, unlike the weaker $\mathrm{C}-\mathrm{Br}$ bond which is broken immediately upon adsorption. ${ }^{59}$ Dechlorination of the precursor molecules thus occurs in a broad temperature interval from 380 to $470 \mathrm{~K}$ as evidenced by a gradual redistribution of the $\mathrm{Cl} 2 \mathrm{p}$ intensity from the high $E_{\mathrm{B}}$ to the low $E_{\mathrm{B}}$ spin doublet. At temperatures beyond $470 \mathrm{~K}$, all carbon-bound $\mathrm{Cl}$ is gone and only chemisorbed $\mathrm{Cl}$ is left on the surface. The reduction of the total $\mathrm{Cl} 2 \mathrm{p}$ photoemission intensity toward the end of the DCBA dechlorination process can be attributed to chlorine desorption at elevated temperatures.

Information about changes in the chemical environment of carbon atoms comes from analysis of the $\mathrm{C} 1 \mathrm{~s}$ spectra acquired at several characteristic annealing temperatures. Analysis of the evolution of the $\mathrm{C} 1 \mathrm{~s}$ line shape for DCBA on $\operatorname{Ag}(111)$ was performed using a peak-fitting procedure that was implemented previously for the investigation of the on-surface transformations of DBBA on $\mathrm{Ag}(111)$ and $\mathrm{Cu}(111)$ surfaces. ${ }^{11,59}$ More details of the fitting procedure are presented in the Supporting Information. The C 1s peak can be fitted by four components, representing four groups of chemically distinct carbon atoms: (i) "graphitic" carbon atoms (C[C3]), bonded to three neighboring carbon atoms; (ii) "edge" carbon atoms $(\mathrm{C}[\mathrm{C} 2 \mathrm{H}])$, bonded to two carbon atoms and one hydrogen atom; (iii) chlorine-bonded carbon atoms $(\mathrm{C}[\mathrm{C} 2 \mathrm{Cl}])$; and (iv) silver-bonded carbon atoms (C[C2Ag]). The schematics showing carbon atoms in different chemical environments are shown in Figure S3. The relative intensities of the components are proportional to the fractions of carbon atoms corresponding to distinctive chemical environments.

Figure $2 \mathrm{~b}$ displays the intensities of the different carbon species upon DCBA deposition and subsequent heating on $\mathrm{Ag}(111)$. The intensities were normalized to the number of carbon atoms in chemically inequivalent positions out of the 28 carbon atoms that make up the molecule or repeating unit. The fitted spectra can be found in Figure S3. The temperature windows for dehalogenation, covalent coupling, and cyclodehydrogenation are clearly observed, indicated by blue, green, and gray colors in Figure $2 \mathrm{~b}$. Dechlorination occurs between 380 and $470 \mathrm{~K}$, in agreement with the $\mathrm{Cl} 2 \mathrm{p}$ data. In the same temperature window, the $\mathrm{C}[\mathrm{C} 3] / \mathrm{C}[\mathrm{C} 2 \mathrm{H}]$ ratio increases from $10: 16$ to $12: 16$. These ratios are consistent with the theoretical $\mathrm{C}[\mathrm{C} 3] / \mathrm{C}[\mathrm{C} 2 \mathrm{H}]$ ratios in pristine DCBA and in covalently coupled polymers, respectively. We conclude that dechlorination and subsequent covalent coupling are correlated.

A tiny fraction of silver-linked carbon atoms (the $\mathrm{C}[\mathrm{C} 2 \mathrm{Ag}]$ state) emerges upon dechlorination at $410 \mathrm{~K}$. Organometallic (OM) chains were reported for DBBA on $\operatorname{Ag}(111) .^{59}$ However, the formation of OM chains would lead to the appearance of at least two carbon atoms in the $\mathrm{C}[\mathrm{C} 2 \mathrm{Ag}]$ state per molecular unit. In the present case of DCBA on $\operatorname{Ag}(111)$, the observed signature of carbon atoms in the $\mathrm{C}[\mathrm{C} 2 \mathrm{Ag}]$ state is 
too small to be explained by the formation of OM structures. For DCBA on $\mathrm{Ag}(111)$, the absence of OM structures from DCBA can be attributed to the high annealing temperatures required for dissociation of the $\mathrm{C}-\mathrm{Cl}$ bond. Indeed, cleavage of the $\mathrm{C}-\mathrm{Ag}$ bond was reported already at $400 \mathrm{~K},{ }^{59}$ which means that most of the $\mathrm{C}-\mathrm{Ag}$ bonds will readily dissociate at temperatures required for dechlorination. Hence, no $\mathrm{OM}$ intermediates are formed, and the molecules tend to link covalently right upon dehalogenation, indicating an Ullmann coupling mechanism. ${ }^{51}$

Figure $2 \mathrm{~b}$ also shows that above $470 \mathrm{~K}$, the fraction of $\mathrm{C}[\mathrm{C} 2 \mathrm{H}]$ sites decreases, accompanied by a proportional growth of the $\mathrm{C}[\mathrm{C} 3]$ site fraction. This is a clear indication of cyclodehydrogenation. The approximate $\mathrm{C}[\mathrm{C} 3] / \mathrm{C}[\mathrm{C} 2 \mathrm{H}]$ ratio increases from $12: 16$ at $470 \mathrm{~K}$ to $22: 6$ at $560 \mathrm{~K}$, surpassing a theoretical $\mathrm{C}[\mathrm{C} 3] / \mathrm{C}[\mathrm{C} 2 \mathrm{H}]$ ratio of $20: 8$ for 7 acGNRs and 3,1-cGNRs. This can be explained by the tendency of $\operatorname{Ag}(111)$ to promote lateral fusion, or crossdehydrogenative coupling, of GNRs. ${ }^{56}$ It is likely that GNRs are formed around 530-540 K, while annealing above $540 \mathrm{~K}$ promotes lateral fusion, increasing the fraction of graphitic carbon atoms even further. The graphitization of molecules upon annealing is also confirmed by angular-dependent NEXAFS spectroscopy measurements (Figure S5).

To generalize our knowledge about the spectroscopy fingerprints for different on-surface reactions of DCBA on coinage metals, we extended our XPS investigations to $\mathrm{Cu}(111)$ and $\mathrm{Au}(111)$ substrates. On these surfaces, DCBA has been reported to give 3,1-chiral GNRs ${ }^{45}$ and poorly ordered bisanthene oligomers, ${ }^{46}$ respectively. The XPS results are discussed in detail in the Supporting Information (Figures S3b,c and S4). In short, our data support the conclusion that surface-assisted dehydrogenative coupling takes place on $\mathrm{Cu}(111)$, irrespective of dehalogenation, leading to the formation of 3,1-cGNR from DBBA, DCBA, and BA. ${ }^{44,45}$ The data on $\mathrm{Au}(111)$ corroborate the picture that the increased dehalogenation temperature in DCBA interferes with the regular Ullmann coupling pathway, and irregular polybisanthenes are formed because of nonselective couplings of planarized molecules. ${ }^{46}$ Importantly, these experiments show that for each surface, the dehalogenation temperature of the chlorinated molecule is higher compared to that of the brominated analogue, which may or may not interfere with the "normal" sequence of on-surface reactions to give well-defined GNRs.

In fact, it is exactly this elevated dehalogenation temperature for the chlorinated precursor that may also underlie the anomalous behavior of DCBA on $\operatorname{Ag}(111)$ : the formation of both 7-acGNR and 3,1-cGNRs. Indeed, if the Ullmann-type coupling is disturbed, a pathway may open for surface-assisted dehydrogenative (non-Ullmann) coupling, resulting in 3,1cGNR in analogous fashion to what was found before on $\mathrm{Cu}(111) \cdot{ }^{42-45}$ Interference of the Ullmann coupling could arise from passivation of bianthryl radicals, for example, through the release of hydrogen atoms on the surface resulting from premature cyclodehydrogenation occurring in some molecules.

SPM Study of the Formation Mechanism. We continue our investigations by returning to SPM imaging of DCBA on $\operatorname{Ag}(111)$, where we use the results of our XPS measurements as a guide to produce various reaction intermediates. Figure 3a,b shows DCBA evaporated onto $\operatorname{Ag}(111)$ at RT. The molecules assemble into extensive patches which resemble

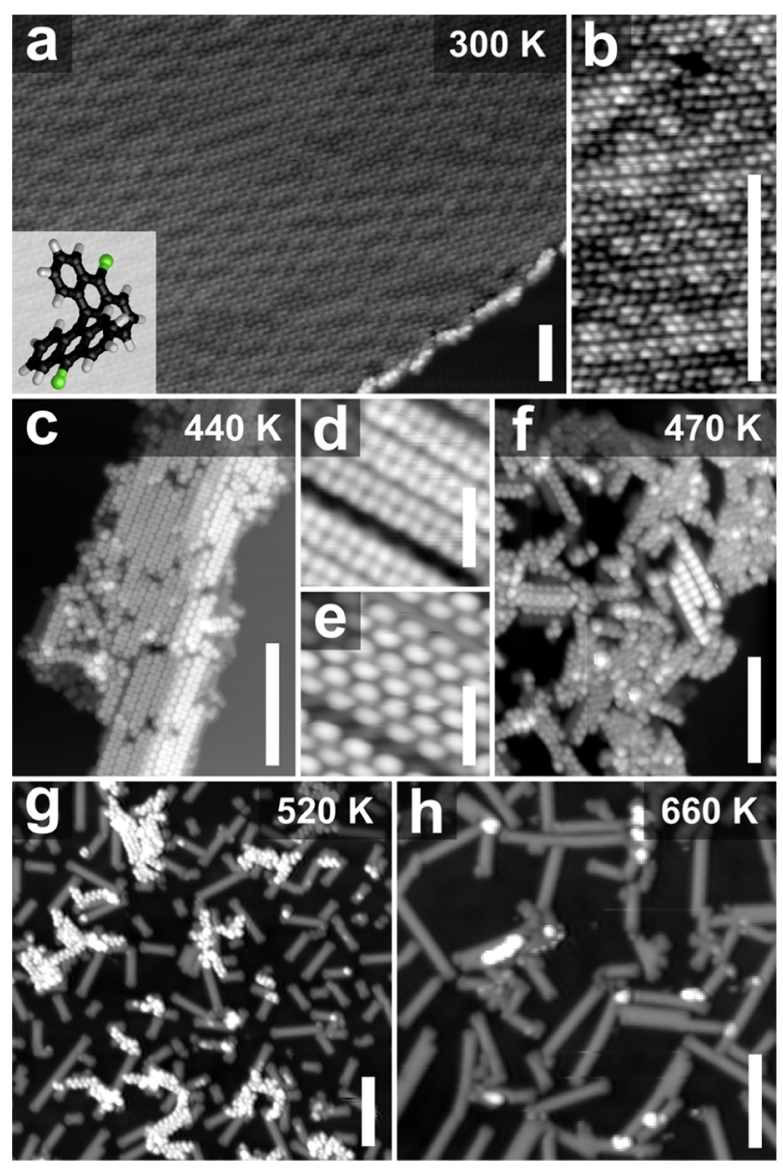

Figure 3. STM scans of DCBA on $\operatorname{Ag}(111)$ at various temperatures. (a) STM scan $(V=-2 \mathrm{~V}, I=20 \mathrm{pA})$ showing assemblies of DCBA as obtained from depositing at RT. (b) Higher-resolution STM scan ( $V$ $=-2 \mathrm{~V}, I=20 \mathrm{pA})$ of the assembly of (a). (c) STM scan $(V=-2 \mathrm{~V}, I$ $=20 \mathrm{pA})$ of the sample of $(\mathrm{a}, \mathrm{b})$ after annealing to $440 \mathrm{~K}$. (d) Higherresolution STM scan $(V=-2 \mathrm{~V}, I=20 \mathrm{pA})$ of the polymers of (c). (e) STM scan of the same region as (d), but at $(V=-1.6 \mathrm{~V}, I=20$ pA). (f) STM scan $(V=-1.2 \mathrm{~V}, I=20 \mathrm{pA})$ after annealing to $470 \mathrm{~K}$. (g) STM scan $(V=-1.6 \mathrm{~V}, I=20 \mathrm{pA})$ after annealing to $520 \mathrm{~K}$. (h) STM scan $(V=-1.6 \mathrm{~V}, I=20 \mathrm{pA})$ after annealing to $660 \mathrm{~K}$. Scale bars in $(\mathrm{d}, \mathrm{e})$ are $2 \mathrm{~nm}$. All other scale bars are $10 \mathrm{~nm}$.

those of the same intact molecule on $\mathrm{Au}(111) .{ }^{46}$ Unlike DBBA, no assembly into chiral "windmill-like" structures is observed. ${ }^{70}$ Heating to $440 \mathrm{~K}$-the dehalogenation temperature as found from XPS - gives the situation shown in Figure 3c. The molecules have assembled into polymeric chains. Although the XPS experiments show that dehalogenation is not yet complete at this temperature, we have not been able to identify any remaining uncoupled molecules on this sample, possibly because of a slight discrepancy in the temperature calibration of the SPM setup with respect to the XPS setup.

We argue that it might not be clear a priori whether the polymers of Figure $3 c$ are the polyanthrylenes that precede the 7-acGNR or the chiral polymer that precede the 3,1-cGNR, especially considering the fact that earlier studies concluded that the two polymers have a very similar appearance in STM. ${ }^{45,56}$ Previous attempts to resolve the type of polymer have made use of nc-AFM, but the nonplanar nature still makes it difficult to unequivocally assign the correct geometry. ${ }^{45}$ However, in our experiments, we found that increasing the negative bias voltage in STM past $-1.8 \mathrm{~V}$ induces an abrupt contrast change in the polymers: scanning at 


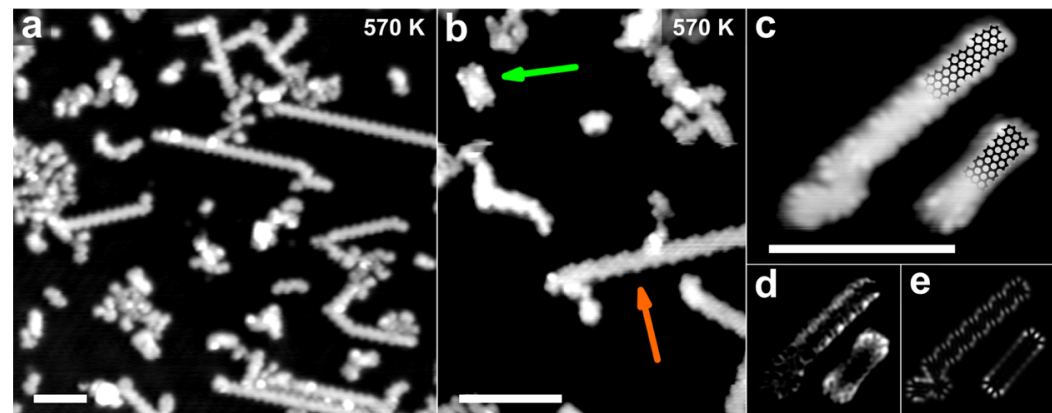

Figure 4. Experiments with codeposited DCBA and DBP. (a) STM scan $(V=-1 \mathrm{~V}, I=20 \mathrm{pA})$ of $\mathrm{Ag}(111)$ onto which a mixture of DCBA and DBP was annealed to $570 \mathrm{~K}$. (b) STM scan $(V=-1 \mathrm{~V}, I=20 \mathrm{pA})$ of the sample of (a) onto which subsequent synthesis with more DCBA was performed. The orange arrow shows a 3,1-cGNR, whereas the green arrow shows a 7-acGNR. (c) STM scan $(V=-1 \mathrm{~V}, I=20 \mathrm{pA})$ of a 7 -acGNR and (imperfect) 3,1-cGNR lying side-by-side, from the sample of (b). The atomic geometry of the two types of nanoribbons is overlaid. (d) Differential conductance map $(V=-0.8 \mathrm{~V}, I=20 \mathrm{pA})$ of the ribbons of $(\mathrm{c})$. (e) Simulated local density of states map corresponding to the ribbons of (c). All scale bars are $5 \mathrm{~nm}$.

Scheme 1. Proposed Reaction Mechanisms for DBBA/DCBA with DBP ${ }^{a}$

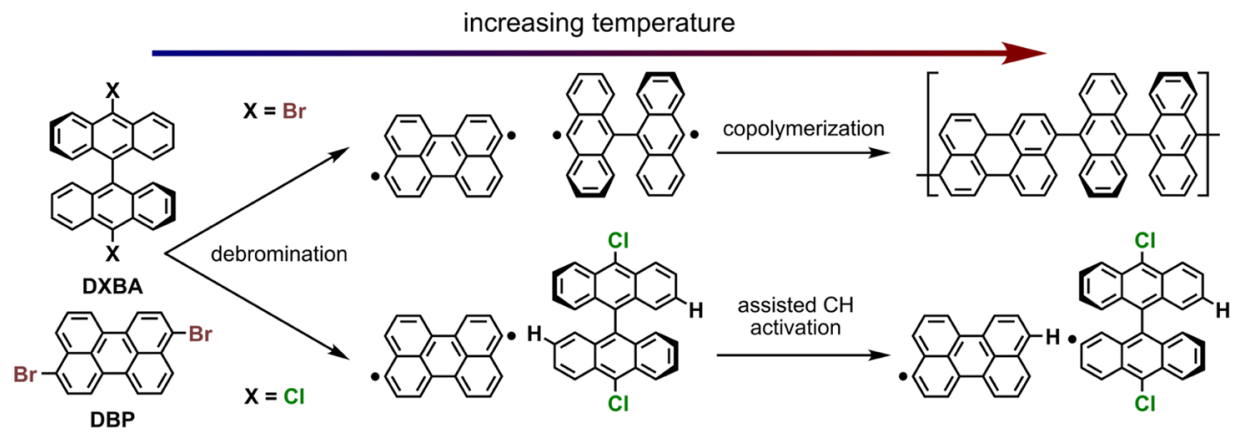

${ }^{a_{\text {Top: }}}$ As shown earlier on $\mathrm{Au}(111)$, using the brominated species, both molecules undergo dehalogenation and subsequent coupling into armchair nanoribbon heterojunctions. ${ }^{27}$ Bottom: Using DBP with DCBA on $\mathrm{Ag}(111)$ gives the situation where at the debromination temperature, only the DBP is dehalogenated. The radicals originating from DBP could induce $\mathrm{CH}$ activation in DCBA, promoting the surface-assisted dehydrogenative coupling into 3,1-cGNRs.

$-2 \mathrm{~V}$ results in images where the polymers have twice as many lobes as at lower absolute bias. This is shown in more detail in Figure 3d,e. Interestingly, this "lobe-doubling" feature enables us to assign the polyanthrylene structure rather than the chiral polymer structure (see Figure S6).

We carried out further gradual heating of these polymers, similar to the procedures used before in the XPS experiments and the SPM experiments resulting in the formation of 7 acGNRs. This results in cyclodehydrogenation, which is found to start at $470 \mathrm{~K}$ (Figure 3f), is in full swing at $520 \mathrm{~K}$ (Figure $3 \mathrm{~g}$ ), and is almost fully completed at $660 \mathrm{~K}$ (Figure $3 \mathrm{~h}$ ). At this highest temperature, a significant number of 14- and 21acGNRs is observed as well. The lateral fusion of 7-acGNRs explains why, in the C 1s XPS spectra, the theoretical C[C3]/ $\mathrm{C}[\mathrm{C} 2 \mathrm{H}]$ ratio of $20: 8$ was surpassed to give a ratio of $22: 6$. Cross-dehydrogenative coupling generates a significantly larger proportion of graphitic carbon atoms compared to what is expected for either the 7-acGNR or the 3,1-cGNR.

Finally, we remark that we attempted to find the hypothetic $\mathrm{OM}$ intermediate that would precede the polymer phase on $\mathrm{Ag}(111)$, in analogy with DBBA. However, no polymers were found below $420 \mathrm{~K}$. This is in agreement with the exceedingly low $\mathrm{C}[\mathrm{C} 2 \mathrm{Ag}]$ intensity in the $\mathrm{C} 1 \mathrm{~s}$ spectra and matches our earlier conclusion that the elevation of the dehalogenation temperature of DCBA relative to that of DBBA causes DCBA to polymerize without adopting the intermediate $O M$ phase on $\operatorname{Ag}(111)$ : it couples covalently right after dechlorination.
DCBA with DBP on Ag(111): Promoting Chiral Nanoribbon Formation. Our previous experiments showed that slow or stepwise annealing of DCBA on $\operatorname{Ag}(111)$ is likely to result in 7-acGNRs, whereas 3,1-cGNRs were obtained when the sample was heated suddenly. This is likely due to the presence of atoms on the surface that block the normal Ullmann coupling pathway. From the XPS experiments, it was deduced that the close temperature proximity of dehalogenation and cyclodehydrogenation could be the underlying cause of the blocking of the Ullmann coupling pathway: molecules undergoing premature cyclodehydrogenation release hydrogen atoms that may passivate the bianthryl radicals. An interesting question that arises is whether it is possible to exploit the sensitivity of the reaction sequence induced by this temperature proximity and thus to control the type of GNR that is obtained.

As shown recently by Liu et al., the presence of coadsorbates can induce substantial changes to an on-surface reaction mechanism, promoting elementary reaction steps that would not occur without their presence. ${ }^{50}$ This result proves that coadsorbed molecular species can have a substantial effect in an on-surface synthesis protocol, even in the form of a catalyst. With this in mind, we decided to perform synthesis with DCBA with coadsorbed 3,9- and 3,10-dibromoperylene (DBP). DBP is known to give five-atom wide armchair nanoribbons on $\mathrm{Au}(111)^{27,38}$ and $\mathrm{Cu}(111){ }^{45} \mathrm{On} \mathrm{Cu}(111)$, two OM intermediates are observed: a "wiggly-chain" 
intermediate consisting of biradical species and a straight-chain intermediate which contains tetraradicals. The transformation from the biradical to tetraradical occurs at the expense of two hydrogen atoms. The nanoribbon formation behavior of DBP on $\operatorname{Ag}(111)$ is outlined in Figure S7, where it is verified that 5acGNRs can indeed be obtained on this surface.

Figure 4a shows the result of on-surface synthesis with both DCBA and DBP on $\operatorname{Ag}(111)$, achieved by depositing both species in an equal amount onto the metal surface followed by slow annealing to $570 \mathrm{~K}$. Despite using the same heating rate that earlier gave rise to 7-acGNRs exclusively, this time we only get the 3,1-cGNR. More precisely, we find that the two molecular species have segregated, with DBP interconnecting to give poorly ordered graphitic structures, and DCBA coupling into 3,1-cGNRs. The long, straight, and mostly defect-free segments of the 3,1-cGNRs suggest a largely unimpeded non-Ullmann-type coupling of the DCBA. Moreover, the chiral nanoribbons make up roughly half of the material on the surface. Because an approximately equal amount of DBP and DCBA was deposited, this suggests that DCBA is transformed predominantly into 3,1-cGNRs rather than into graphitic structures. Thus, the yield of 3,1-cGNR formation in this experiment is very high: much higher than what was achieved without DBP. The segregation behavior of DBP and DCBA and resultant coupling into 3,1-cGNR is in large contrast with the unimpeded copolymerization of DBP and DBBA into acGNR heterojunctions on $\mathrm{Au}(111)^{27}$ (see Scheme 1: top) and the incompatible coupling behavior of DBP with DBBA on $\mathrm{Cu}(111)$, where in the last case, the DBP actually interferes with the formation of 3,1-cGNRs instead of promoting it (see Figure S8). We have therefore found that the presence of DBP promotes the surface-assisted coupling mechanism of DCBA to 3,1-cGNR on $\operatorname{Ag}(111)$.

We have come up with two possible explanations for the effect of DBP on the reaction mechanism of DCBA on $\operatorname{Ag}(111)$. First, as shown in Scheme 1, the presence of both a brominated and a chlorinated molecular species gives rise to a temperature window where only the bromine-bearing precursor has dehalogenated. Because DBP has a planar shape, its radical could approach the $\mathrm{DCBA} \mathrm{CH}(2)$ position and promote its activation. This explanation puts DBP into the role of the activator for surface-assisted dehydrogenative coupling. Subsequent coupling of thus-obtained radicals of DCBA yields 3,1-cGNRs. This scenario also suggests that DBP radicals could be prematurely passivated in the process of activating DCBA. Coupling of partially passivated DBP molecules is likely to be nonselective, explaining why DBP forms poorly ordered graphitic structures. An alternative explanation puts DBP into the role of the hydrogen source. Because the OM intermediate found for pure DBP at $450 \mathrm{~K}$ is reminiscent of the tetraradical species found earlier for DBP on $\mathrm{Cu}(111)$ (see Figure S7), DBP may have already undergone dehydrogenative coupling at the DCBA dechlorination temperature. The hydrogen thus released could inhibit Ullmann coupling by passivating dechlorinated DCBA radical species. Regardless of which explanation is correct, both hypotheses rely on the fact that the increased dehalogenation temperature of DCBA gives rise to the possibility of switching from the Ullmann coupling mechanism to surface-assisted dehydrogenation.

Finally, having acquired a sample with 3,1-cGNR, we deposited more DCBA and repeated the heating procedure. Because all interfering molecular species present on the surface have already coupled, no promotion of the surface-assisted coupling pathway should be expected. Indeed, this time, several short 7-acGNRs were obtained, next to the already existing chiral nanoribbons, as shown in Figure 4b. To unequivocally prove the nature of the two different types of nanoribbons, we performed differential conductance mapping on a 7-acGNR and 3,1-cGNR lying next to each other, as can be seen in Figure 4c,d. Indeed, a map at low bias voltage shows end-localized states in the 7-acGNR and edge-localized states in the 3,1-cGNR (Figure 4d), in agreement with a simulated map from a simple tight-binding calculation (Figure $4 \mathrm{e}$ ). This experiment finalizes our hypothesis that the 3,1-cGNR can be obtained by blocking the Ullmann coupling pathway or promotion of the surface-assisted dehydrogenative coupling pathway.

\section{CONCLUSIONS}

In conclusion, we present a systematic study of the behavior of DCBA on $\operatorname{Ag}(111)$. Our STM measurements enabled us to disclose the nature of the different nanoribbons as well as that of the polymeric intermediate. We demonstrate that both the 7-acGNR and the 3,1-cGNR can be obtained: the first combination of the molecule and metal surface that allows for the synthesis of two different kinds of GNRs side-by-side. This means that the structure of GNRs is in some cases determined by more subtle factors than even the molecular structure and the surface.

Our XPS study allowed us to get deeper insights into the behavior of brominated and chlorinated bianthryls on the coinage metal surfaces and get information about the activation temperatures of on-surface reactions. For DCBA on $\mathrm{Au}(111)$, dehalogenation takes place after cyclodehydrogenation, resulting in the formation of poorly ordered bisanthene polymers as observed previously in STM. ${ }^{46}$ In turn, the more reactive $\operatorname{Ag}(111)$ surface decreases the dechlorination temperature with respect to that on $\mathrm{Au}(111)$ by virtue of its larger reactivity. As a result, the formation of well-defined nanoribbons can take place from DCBA, and all intermediates could be resolved in STM measurements. $\mathrm{Cu}(111)$ blocks the Ullmann coupling mechanism, promoting surface-assisted dehydrogenative coupling regardless of the halogen. However, for the (111) surfaces of each coinage metal, we find that dechlorination takes place at higher temperature than debromination, which can crucially affect the reaction mechanism. ${ }^{57}$ In fact, the significantly higher dehalogenation temperature of DCBA with respect to its brominated analogue brings the dehalogenation temperature close to the onset for the surface-assisted dehydrogenation.

The small energetic separation of the dehalogenation and dehydrogenation temperatures for DCBA in comparison with those for DBBA may result in an increased likelihood of switching to non-Ullmann, surface-assisted dehydrogenative coupling. It was suggested that the presence of the reaction side products (most likely hydrogen) or coadsorbates interfering with Ullmann coupling can make the alternative reaction pathway possible. This hypothesis was verified in an experiment where we promoted the formation of 3,1-cGNRs by using coadsorbed DBP. DBP affects the reaction sequence in such a way that the surface-assisted dehydrogenative coupling mechanism is promoted. Rather than giving a copolymer as on $\mathrm{Au}(111)^{27}$ or $\mathrm{Cu}(111), 3,1$-cGNRs were obtained, even when using the gradual heating procedure that earlier gave rise to the 7-acGNR exclusively. 
We conclude that the unique match of intermediate reactivity of the $\operatorname{Ag}(111)$ surface and the relatively high dechlorination barrier of DCBA enables the surface-assisted dehydrogenative coupling pathway, explaining why sometimes DCBA couples into 3,1-cGNR. Therefore, DCBA on Ag(111) is a combination of molecule and metal surface that can give rise to either the 7-acGNR or the 3,1-cGNR depending on the reaction parameters and the presence of co-adsorbed species. The processes identified in our combined SPM and XPS studies reveal design rules for on-surface syntheses involving molecules on metals and will aid in future strategies for bottom-up synthesis of GNRs and other molecular nanostructures.

\section{ASSOCIATED CONTENT}

\section{S Supporting Information}

The Supporting Information is available free of charge on the ACS Publications website at DOI: 10.1021/acs.jpcc.8b12209.

Additional experimental details and data (PDF)

\section{AUTHOR INFORMATION}

\section{Corresponding Authors}

*E-mail: jacobse@berkeley.edu (P.H.J.).

*E-mail: konstantin.simonov@physics.uu.se (K.A.S.).

\section{ORCID $\odot$}

Peter H. Jacobse: 0000-0002-0777-6050

Anders Sandell: 0000-0002-6120-7502

\section{Author Contributions}

P.H.J. and K.A.S. conceived the experiments. The SPM experiments were performed by P.H.J. and M.J.J.M. The XPS and NEXAFS measurements were conducted by K.A.S., P.H.J., M.J.J.M., G.I.S., A.V.G., and A.B.P. Tight-binding calculations were performed by P.H.J. The data were evaluated by all the authors, and all the authors contributed to the manuscript.

\section{Notes}

The authors declare no competing financial interest.

\section{ACKNOWLEDGMENTS}

The authors are grateful for the financial support from the Netherlands Organization for Scientific Research (NWO), the Swedish Research Council, the Swedish Energy Agency (STEM), the Carl Trygger foundation for scientific research, the European Research Council under the European Union's Seventh Framework Program (FP7/2007-2013)/ERC grant agreement no. [321319], Knut and Alice Wallenberg Foundation, and the Russian Foundation for Basic Research (grant no. 15-02-06369). The authors thank HZB for the allocation of beamtime at BESSY II and the HE-SGM staff for the support during the experiments.

\section{REFERENCES}

(1) Son, Y.-W.; Cohen, M. L.; Louie, S. G. Energy Gaps in Graphene Nanoribbons. Phys. Rev. Lett. 2006, 97, 216803.

(2) Wakabayashi, K.; Sasaki, K.-I.; Nakanishi, T.; Enoki, T. Electronic States of Graphene Nanoribbons and Analytical Solutions. Sci. Technol. Adv. Mater. 2010, 11, 054504.

(3) Barone, V.; Hod, O.; Scuseria, G. E. Electronic Structure and Stability of Semiconducting Graphene Nanoribbons. Nano Lett. 2006, 6, 2748-2754.

(4) Yazyev, O. V. A Guide to the Design of Electronic Properties of Graphene Nanoribbons. Acc. Chem. Res. 2013, 46, 2319-2328.
(5) Raza, H.; Kan, E. C. Armchair Graphene Nanoribbons: Electronic Structure and Electric-Field Modulation. Phys. Rev. B: Condens. Matter Mater. Phys. 2008, 77, 245434.

(6) Saroka, V. A.; Batrakov, K. G.; Chernozatonskii, L. A. EdgeModified Zigzag-Shaped Graphene Nanoribbons: Structure and Electronic Properties. Phys. Solid State 2014, 56, 2135-2145.

(7) Han, M. Y.; Özyilmaz, B.; Zhang, Y.; Kim, P. Energy Band-Gap Engineering of Graphene Nanoribbons. Phys. Rev. Lett. 2007, 98, 206805.

(8) Yang, L.; Park, C.-H.; Son, Y.-W.; Cohen, M. L.; Louie, S. G. Quasiparticle Energies and Band Gaps in Graphene Nanoribbons. Phys. Rev. Lett. 2007, 99, 186801.

(9) Ma, L.; Wang, J.; Ding, F. Recent Progress and Challenges in Graphene Nanoribbon Synthesis. ChemPhysChem 2013, 14, 47-54.

(10) Cai, J.; Ruffieux, P.; Jaafar, R.; Bieri, M.; Braun, T.; Blankenburg, S.; Muoth, M.; Seitsonen, A. P.; Saleh, M.; Feng, X.; et al. Atomically Precise Bottom-up Fabrication of Graphene Nanoribbons. Nature 2010, 466, 470-473.

(11) Simonov, K. A.; Vinogradov, N. A.; Vinogradov, A. S.; Generalov, A. V.; Zagrebina, E. M.; Mårtensson, N.; Cafolla, A. A.; Carpy, T.; Cunniffe, J. P.; Preobrajenski, A. B. Effect of Substrate Chemistry on the Bottom-up Fabrication of Graphene Nanoribbons: Combined Core-Level Spectroscopy and STM Study. J. Phys. Chem. C 2014, 118, 12532-12540.

(12) Simonov, K. A.; Vinogradov, N. A.; Vinogradov, A. S.; Generalov, A. V.; Svirskiy, G. I.; Cafolla, A. A.; Mårtensson, N.; Preobrajenski, A. B. Effect of Electron Injection in Copper-Contacted Graphene Nanoribbons. Nano Res. 2016, 9, 2735-2746.

(13) James, D. K.; Tour, J. M. The Chemical Synthesis of Graphene Nanoribbons-A Tutorial Review. Macromol. Chem. Phys. 2012, 213, $1033-1050$

(14) Cai, J.; Pignedoli, C. A.; Talirz, L.; Ruffieux, P.; Söde, H.; Liang, L.; Meunier, V.; Berger, R.; Li, R.; Feng, X.; et al. Graphene Nanoribbon Heterojunctions. Nat. Nanotechnol. 2014, 9, 896-900.

(15) Chen, Y.-C.; De Oteyza, D. G.; Pedramrazi, Z.; Chen, C.; Fischer, F. R.; Crommie, M. F. Tuning the Band Gap of Graphene Nanoribbons Synthesized from Molecular Precursors. ACS Nano 2013, 7, 6123-6128.

(16) Ruffieux, P.; Wang, S.; Yang, B.; Sánchez-Sánchez, C.; Liu, J.; Dienel, T.; Talirz, L.; Shinde, P.; Pignedoli, C. A.; Passerone, D.; et al. On-Surface Synthesis of Graphene Nanoribbons with Zigzag Edge Topology. Nature 2016, 531, 489-492.

(17) Xu, W.; Lee, T.-W. Recent Progress in Fabrication Techniques of Graphene Nanoribbons. Mater. Horiz. 2016, 3, 186-207.

(18) Bronner, C.; Durr, R. A.; Rizzo, D. J.; Lee, Y.-L.; Marangoni, T.; Kalayjian, A. M.; Rodriguez, H.; Zhao, W.; Louie, S. G.; Fischer, F. R.; et al. Hierarchical On-Surface Synthesis of Graphene Nanoribbon Heterojunctions. ACS Nano 2018, 12, 2193-2200.

(19) Bronner, C.; Stremlau, S.; Gille, M.; Brauße, F.; Haase, A.; Hecht, S.; Tegeder, P. Aligning the Band Gap of Graphene Nanoribbons by Monomer Doping. Angew. Chem., Int. Ed. 2013, $52,4422-4425$.

(20) Chen, Y.-C.; Cao, T.; Chen, C.; Pedramrazi, Z.; Haberer, D.; De Oteyza, D. G.; Fischer, F. R.; Louie, S. G.; Crommie, M. F. Molecular Bandgap Engineering of Bottom-up Synthesized Graphene Nanoribbon Heterojunctions. Nat. Nanotechnol. 2015, 10, 156-160.

(21) Marconcini, P.; Cresti, A.; Triozon, F.; Fiori, G.; Biel, B.; Niquet, Y.-M.; MacUcci, M.; Roche, S. Atomistic Boron-Doped Graphene Field-Effect Transistors: A Route toward Unipolar Characteristics. ACS Nano 2012, 6, 7942-7947.

(22) Mohamadpour, H.; Asgari, A. Graphene Nanoribbon Tunneling Field Effect Transistors. Phys. E 2012, 46, 270-273.

(23) Huang, B.; Yan, Q.-m.; Li, Z.-y.; Duan, W.-h. Towards Graphene Nanoribbon-Based Electronics. Front. Phys. China 2009, 4, 269-279.

(24) Bennett, P. B.; Pedramrazi, Z.; Madani, A.; Chen, Y.-C.; De Oteyza, D. G.; Chen, C.; Fischer, F. R.; Crommie, M. F.; Bokor, J. Bottom-up Graphene Nanoribbon Field-Effect Transistors. Appl. Phys. Lett. 2013, 103, 253114. 
(25) Wang, X.; Ouyang, Y.; Jiao, L.; Wang, H.; Xie, L.; Wu, J.; Guo, J.; Dai, H. Graphene Nanoribbons with Smooth Edges Behave as Quantum Wires. Nat. Nanotechnol. 2011, 6, 563-567.

(26) Llinas, J. P.; Fairbrother, A.; Borin Barin, G.; Shi, W.; Lee, K.; Wu, S.; Yong Choi, B.; Braganza, R.; Lear, J.; Kau, N.; et al. ShortChannel Field-Effect Transistors with 9-Atom and 13-Atom Wide Graphene Nanoribbons. Nat. Commun. 2017, 8, 633.

(27) Jacobse, P. H.; Kimouche, A.; Gebraad, T.; Ervasti, M. M.; Thijssen, J. M.; Liljeroth, P.; Swart, I. Electronic Components Embedded in a Single Graphene Nanoribbon. Nat. Commun. 2017, 8, 119.

(28) Jacobse, P. H.; Mangnus, M. J. J.; Zevenhuizen, S. J. M.; Swart, I. Mapping the Conductance of Electronically Decoupled Graphene Nanoribbons. ACS Nano 2018, 12, 7048-7056.

(29) Fang, T.; Konar, A.; Xing, H.; Jena, D. Mobility in Semiconducting Graphene Nanoribbons: Phonon, Impurity, and Edge Roughness Scattering. Phys. Rev. B: Condens. Matter Mater. Phys. 2008, 78, 205403.

(30) Evaldsson, M.; Zozoulenko, I. V.; Xu, H.; Heinzel, T. EdgeDisorder-Induced Anderson Localization and Conduction Gap in Graphene Nanoribbons. Phys. Rev. B: Condens. Matter Mater. Phys. 2008, 78, 161407.

(31) Libisch, F.; Rotter, S.; Burgdörfer, J. Coherent Transport through Graphene Nanoribbons in the Presence of Edge Disorder. New J. Phys. 2012, 14, 123006.

(32) Nguyen, G. D.; Toma, F. M.; Cao, T.; Pedramrazi, Z.; Chen, C.; Rizzo, D. J.; Joshi, T.; Bronner, C.; Chen, Y.-C.; Favaro, M.; et al. Bottom-Up Synthesis of $N=13$ Sulfur-Doped Graphene Nanoribbons. J. Phys. Chem. C 2016, 120, 2684-2687.

(33) Cloke, R. R.; Marangoni, T.; Nguyen, G. D.; Joshi, T.; Rizzo, D. J.; Bronner, C.; Cao, T.; Louie, S. G.; Crommie, M. F.; Fischer, F. R. Site-Specific Substitutional Boron Doping of Semiconducting Armchair Graphene Nanoribbons. J. Am. Chem. Soc. 2015, 137, 88728875.

(34) Kawai, S.; Saito, S.; Osumi, S.; Yamaguchi, S.; Foster, A. S.; Spijker, P.; Meyer, E. Atomically Controlled Substitutional BoronDoping of Graphene Nanoribbons. Nat. Commun. 2015, 6, 8098.

(35) Nguyen, G. D.; Tsai, H.-Z.; Omrani, A. A.; Marangoni, T.; Wu, M.; Rizzo, D. J.; Rodgers, G. F.; Cloke, R. R.; Durr, R. A.; Sakai, Y.; et al. Atomically Precise Graphene Nanoribbon Heterojunctions from a Single Molecular Precursor. Nat. Nanotechnol. 2017, 12, 10771082.

(36) Narita, A.; Wang, X.-Y.; Feng, X.; Müllen, K. New Advances in Nanographene Chemistry. Chem. Soc. Rev. 2015, 44, 6616-6643.

(37) Talirz, L.; Söde, H.; Dumslaff, T.; Wang, S.; Sanchez-Valencia, J. R.; Liu, J.; Shinde, P.; Pignedoli, C. A.; Liang, L.; Meunier, V.; et al. On-Surface Synthesis and Characterization of 9-Atom Wide Armchair Graphene Nanoribbons. ACS Nano 2017, 11, 1380-1388.

(38) Kimouche, A.; Ervasti, M. M.; Drost, R.; Halonen, S.; Harju, A.; Joensuu, P. M.; Sainio, J.; Liljeroth, P. Ultra-Narrow Metallic Armchair Graphene Nanoribbons. Nat. Commun. 2015, 6, 10177.

(39) Zhang, H.; Lin, H.; Sun, K.; Chen, L.; Zagranyarski, Y.; Aghdassi, N.; Duhm, S.; Li, Q.; Zhong, D.; Li, Y.; et al. On-Surface Synthesis of Rylene-Type Graphene Nanoribbons. J. Am. Chem. Soc. 2015, 137, 4022-4025.

(40) Celis, A.; Nair, M. N.; Taleb-Ibrahimi, A.; Conrad, E. H.; Berger, C.; de Heer, W. A.; Tejeda, A. Graphene Nanoribbons: Fabrication, Properties and Devices. J. Phys. D: Appl. Phys. 2016, 49, 143001.

(41) Narita, A.; Feng, X.; Müllen, K. Bottom-up Synthesis of Chemically Precise Graphene Nanoribbons. Chem. Rec. 2015, 15, 295-309.

(42) Han, P.; Akagi, K.; Federici Canova, F.; Mutoh, H.; Shiraki, S.; Iwaya, K.; Weiss, P. S.; Asao, N.; Hitosugi, T. Bottom-up GrapheneNanoribbon Fabrication Reveals Chiral Edges and Enantioselectivity. ACS Nano 2014, 8, 9181-9187.

(43) Han, P.; Akagi, K.; Federici Canova, F.; Shimizu, R.; Oguchi, H.; Shiraki, S.; Weiss, P. S.; Asao, N.; Hitosugi, T. Self-Assembly
Strategy for Fabricating Connected Graphene Nanoribbons. ACS Nano 2015, 9, 12035-12044.

(44) Sánchez-Sánchez, C.; Dienel, T.; Deniz, O.; Ruffieux, P.; Berger, R.; Feng, X.; Müllen, K.; Fasel, R. Purely Armchair or Partially Chiral: Noncontact Atomic Force Microscopy Characterization of Dibromo-Bianthryl-Based Graphene Nanoribbons Grown on $\mathrm{Cu}(111)$. ACS Nano 2016, 10, 8006-8011.

(45) Schulz, F.; Jacobse, P. H.; Canova, F. F.; Van Der Lit, J.; Gao, D. Z.; Van Den Hoogenband, A.; Han, P.; Klein Gebbink, R. J. M.; Moret, M.-E.; Joensuu, P. M.; et al. Precursor Geometry Determines the Growth Mechanism in Graphene Nanoribbons. J. Phys. Chem. C 2017, 121, 2896-2904.

(46) Jacobse, P. H.; van den Hoogenband, A.; Moret, M.-E.; Klein Gebbink, R. J. M.; Swart, I. Aryl Radical Geometry Determines Nanographene Formation on $\mathrm{Au}(111)$. Angew. Chem., Int. Ed. 2016, $55,13052-13055$.

(47) Kittelmann, M.; Nimmrich, M.; Lindner, R.; Gourdon, A.; Kühnle, A. Sequential and Site-Specific on-Surface Synthesis on a Bulk Insulator. ACS Nano 2013, 7, 5614-5620.

(48) Kittelmann, M.; Rahe, P.; Nimmrich, M.; Hauke, C. M.; Gourdon, A.; Kühnle, A. On-Surface Covalent Linking of Organic Building Blocks on a Bulk Insulator. ACS Nano 2011, 5, 8420-8425.

(49) Shi, K. J.; Zhang, X.; Shu, C. H.; Li, D. Y.; Wu, X. Y.; Liu, P. N. Ullmann Coupling Reaction of Aryl Chlorides on $\mathrm{Au}(111)$ Using Dosed $\mathrm{Cu}$ as a Catalyst and the Programmed Growth of 2D Covalent Organic Frameworks. Chem. Commun. 2016, 52, 8726-8729.

(50) Liu, M.; Chen, S.; Li, T.; Wang, J.; Zhong, D. Tuning OnSurface Synthesis of Graphene Nanoribbons by Noncovalent Intermolecular Interactions. J. Phys. Chem. C 2018, 122, 2441524420.

(51) Zhou, X.; Bebensee, F.; Shen, Q.; Bebensee, R.; Cheng, F.; He, Y.; Su, H.; Chen, W.; Xu, G. Q.; Besenbacher, F.; et al. On-Surface Synthesis Approach to Preparing One-Dimensional Organometallic and Poly-p-Phenylene Chains. Mater. Chem. Front. 2017, 1, 119-127.

(52) Jarvis, S. Resolving Intra- and Inter-Molecular Structure with Non-Contact Atomic Force Microscopy. Int. J. Mol. Sci. 2015, 16, 19936-19959.

(53) Jacobse, P.; Moret, M.-E.; Klein Gebbink, R.; Swart, I. Tracking On-Surface Chemistry with Atomic Precision. Synlett 2017, 28, 2509-2516

(54) Batra, A.; Cvetko, D.; Kladnik, G.; Adak, O.; Cardoso, C.; Ferretti, A.; Prezzi, D.; Molinari, E.; Morgante, A.; Venkataraman, L. Probing the Mechanism for Graphene Nanoribbon Formation on Gold Surfaces through X-Ray Spectroscopy. Chem. Sci. 2014, 5, 4419-4423.

(55) Massimi, L.; Ourdjini, O.; Lafferentz, L.; Koch, M.; Grill, L.; Cavaliere, E.; Gavioli, L.; Cardoso, C.; Prezzi, D.; Molinari, E.; et al. Surface-Assisted Reactions toward Formation of Graphene Nanoribbons on $\mathrm{Au}(110)$ Surface. J. Phys. Chem. C 2015, 119, 2427-2437.

(56) Simonov, K. A.; Vinogradov, N. A.; Vinogradov, A. S.; Generalov, A. V.; Zagrebina, E. M.; Svirskiy, G. I.; Cafolla, A. A.; Carpy, T.; Cunniffe, J. P.; Taketsugu, T.; et al. From Graphene Nanoribbons on $\mathrm{Cu}(111)$ to Nanographene on $\mathrm{Cu}(110)$ : Critical Role of Substrate Structure in the Bottom-Up Fabrication Strategy. ACS Nano 2015, 9, 8997-9011.

(57) Di Giovannantonio, M.; Deniz, O.; Urgel, J. I.; Widmer, R.; Dienel, T.; Stolz, S.; Sánchez-Sánchez, C.; Muntwiler, M.; Dumslaff, T.; Berger, R.; et al. On-Surface Growth Dynamics of Graphene Nanoribbons: The Role of Halogen Functionalization. ACS Nano 2018, 12, 74-81.

(58) Huang, H.; Wei, D.; Sun, J.; Wong, S. L.; Feng, Y. P.; Neto, A. H. C.; Wee, A. T. S. Spatially Resolved Electronic Structures of Atomically Precise Armchair Graphene Nanoribbons. Sci. Rep. 2012, 2, 983.

(59) Simonov, K. A.; Generalov, A. V.; Vinogradov, A. S.; Svirskiy, G. I.; Cafolla, A. A.; McGuinness, C.; Taketsugu, T.; Lyalin, A.; Mårtensson, N.; Preobrajenski, A. B. Synthesis of Armchair Graphene Nanoribbons from the 10,10'-Dibromo-9,9'-Bianthracene Molecules 
on $\operatorname{Ag}(111)$ : The Role of Organometallic Intermediates. Sci. Rep. 2018, 8, 3506.

(60) van der Lit, J.; Boneschanscher, M. P.; Vanmaekelbergh, D.; Ijäs, M.; Uppstu, A.; Ervasti, M.; Harju, A.; Liljeroth, P.; Swart, I. Suppression of Electron-Vibron Coupling in Graphene Nanoribbons Contacted via a Single Atom. Nat. Commun. 2013, 4, 2023.

(61) Gross, L.; Mohn, F.; Moll, N.; Liljeroth, P.; Meyer, G. The Chemical Structure of a Molecule Resolved by Atomic Force Microscopy. Science 2009, 325, 1110-1114.

(62) Moulder, J. F.; Chastain, J. Handbook of X-ray Photoelectron Spectroscopy: A Reference Book of Standard Spectra for Identification and Interpretation of XPS Data; Perkin-Elmer Corporation Physical Electronics Division: Eden Prairie, 1992.

(63) IGOR Pro. https://www.wavemetrics.com/project/XPStools (accessed Aug 1, 2018).

(64) Koch, M.; Ample, F.; Joachim, C.; Grill, L. Voltage-Dependent Conductance of a Single Graphene Nanoribbon. Nat. Nanotechnol. 2012, 7, 713-717.

(65) Ijäs, M.; Ervasti, M.; Uppstu, A.; Liljeroth, P.; van der Lit, J.; Swart, I.; Harju, A. Electronic States in Finite Graphene Nanoribbons: Effect of Charging and Defects. Phys. Rev. B: Condens. Matter Mater. Phys. 2013, 88, 75429.

(66) Fujita, M.; Wakabayashi, K.; Nakada, K.; Kusakabe, K. Peculiar Localized State at Zigzag Graphite Edge. J. Phys. Soc. Jpn. 1996, 65, 1920-1923.

(67) Talirz, L.; Söde, H.; Cai, J.; Ruffieux, P.; Blankenburg, S.; Jafaar, R.; Berger, R.; Feng, X.; Müllen, K.; Passerone, D.; et al. Termini of Bottom-up Fabricated Graphene Nanoribbons. J. Am. Chem. Soc. 2013, 135, 2060-2063.

(68) Wang, S.; Talirz, L.; Pignedoli, C. A.; Feng, X.; Müllen, K.; Fasel, R.; Ruffieux, P. Giant Edge State Splitting at Atomically Precise Graphene Zigzag Edges. Nat. Commun. 2016, 7, 11507.

(69) Han, P.; Akagi, K.; Federici Canova, F.; Mutoh, H.; Shiraki, S.; Iwaya, K.; Weiss, P. S.; Asao, N.; Hitosugi, T. Reply to "Comment on 'Bottom-Up Graphene-Nanoribbon Fabrication Reveals Chiral Edges and Enantioselectivity". ACS Nano 2015, 9, 3404-3405.

(70) Shen, Y.; Tian, G.; Huang, H.; He, Y.; Xie, Q.; Song, F.; Lu, Y.; Wang, P.; Gao, Y. Chiral Self-Assembly of Nonplanar 10,10'Dibromo-9,9'-bianthryl Molecules on $\operatorname{Ag}(111)$. Langmuir 2017, 33, 2993-2999.

(71) Björk, J.; Stafström, S.; Hanke, F. Zipping up: Cooperativity Drives the Synthesis of Graphene Nanoribbons. J. Am. Chem. Soc. 2011, 133, 14884-14887. 